\title{
Sistemas de acceso y consulta en los diarios digitales españoles
}

\author{
María Victoria Nuño Moral
}

Artículo recibido:

1 de agosto de 2012.

Artículo aceptado:

7 de agosto de 2013.

\section{Resumen}

Se realiza el análisis descriptivo de las formas de acceso y sistemas de consulta con que cuenta el usuario en los diarios digitales españoles desde una doble perspectiva: por un lado señalar cuáles son las formas de acceso y la tipología documental o formatos que alberga cada uno de los sitios web analizados y, por el otro, exponer las características que presentan los respectivos sistemas de búsqueda. Para su realización se ha tomado como referencia la propuesta realizada por Guallar y Abadal (2009) y se ha elaborado una plantilla de trabajo basada en la evaluación descriptiva y comparativa de la muestra seleccionada.

* Universidad de Extremadura, España. mvnunmor@alcazaba.unex.es

INVESTIGACIÓN BIBLIOTECOLÓGICA, Vol. 28, Núm.62, enero/abril, 2014, México, ISSN 0187-358X. pp. 81-99 
Palabras clave: Sistemas de recuperación; Periódicos digitales; Recursos multimedia; Archivos digitales.

\section{Abstract}

Access and retrieval systems in Spanish-language digital newspapers

María-Victoria Nuño-Moral

Researchers offer a descriptive analysis of search tools access paths available to readers of Spanish online newspapers. A twin approach is used: the first examines website in terms of access tool, their typology and the format; while the second approach provides a description of web search systems. The proposal put forth by Guallar and Abadal (2009) serves as a methodological framework of a working template for making descriptive and comparative evaluations of the sample under study.

Keywords: Information retrieval systems; Digital newspapers; Multimedia resources; Digital files.

\section{INTRODUCCIÓN}

T os contenidos que albergan los medios de comunicación en Internet se Lhan convertido en un recurso trascendental, no sólo por su valor informativo sino también por su carácter patrimonial. Actualmente, los medios de comunicación digitales se consideran una fuente de información inestimable, desde un punto de vista general y no sólo periodístico, cuya unicidad en muchas ocasiones les confiere un valor incalculable.

Los diarios digitales son los que, desde su aparición en Internet, han marcado la pauta en el desarrollo de los medios de comunicación. Y son sin duda los que han sufrido un mayor cambio respecto a su antecesor, el formato impreso. De hecho, es ya una práctica habitual encontrar videos, audios, gráficos interactivos o galerías fotográficas como parte de la información que se le ofrece al usuario.

Todo ello ha sido motivado no sólo por el desarrollo tecnológico sino también por la competencia, tanto informativa como empresarial, así como por el 
carácter novedoso y exhaustivo que acompaña a las nuevas demandas formuladas por los usuarios.

En el medio digital las noticias han dejado de ser un mero texto explicativo del hecho en sí, acompañado en ocasiones de fotografía. En la actualidad la información viene custodiada por otros formatos (videos, audios, etc.) que en ocasiones no sólo complementan la información vertida por el texto sino que adoptan un carácter autónomo, sin ningún tipo de dependencia necesaria para su comprensión.

Y como consecuencia, un aspecto esencial es poder acceder y consultar todo ese material, especialmente aquél que no obtenemos en papel mediante la compra diaria. Las fórmulas de acceso son diversas. En el estudio realizado se observa que van desde el método tradicional heredado del formato impreso, basado en secciones, hasta el análisis, las estadísticas de valoración y las prácticas realizadas por los usuarios.

Actualmente son numerosos los trabajos que se han realizado sobre la presencia de los medios de comunicación en Internet y, de manera específica, aquellos que se centran en la prensa digital. Cabe citar monografías en las que se exponen aspectos generales, formales, históricos y funcionales que han revolucionado el modus operandi del periodismo. Entre ellas, destacan las realizadas por autores como Álvarez Marcos y Parra Valcarce (2004); Salaverría (2005); García Jiménez y Rupérez Rubio (2007) o Palacios y Díaz Noci (2009). Se subrayan, igualmente, los estudios llevados a cabo por Pareja Pérez $(2003,2006)$ donde el autor alude a las características de este nuevo medio de comunicación que se cifra en seis aspectos: interactividad, inmediatez, espacialidad indefinida, multimedia o multiformato, funcionalidad y personalización. Además, en el segundo de ellos elabora una guía de recursos comunicativos digitales.

Junto a estos análisis existen otros que observan este tema bajo perspectivas más particulares y especializadas que han permitido saber cuál ha sido la evolución y la situación actual en la que nos encontramos dentro del terreno del ciberperiodismo, marco en el que se encuadra el artículo presente. De especial interés se consideran los artículos que se comentan a continuación.

Luján Zavala (2003) centra su atención en cómo la interactividad, y como consecuencia la aparición de los blogs o weblogs como sección integrada en los sitios web informativos, ha modificado el papel que juega el usuario en la labor informativa actual. Ese impacto de la interactividad es analizado por Parra et al. (2008), haciendo especial hincapié en la rentabilidad económica y empresarial que ello supone ante los nuevos servicios que se ofrecen a los ciudadanos. Esa visión económica también se advierte en el trabajo de Marcos Recio (2005). 
Por su parte, en el IV Congreso Online del Observatorio para la Cibersociedad, Martín Herrera (2010) y García-Alonso Montoya (2010) revisan el escenario y las novedosas herramientas con las que cuenta el profesional para adecuar su trabajo a ese entorno digital y su repercusión laboral, todo ello con el objeto de conseguir la máxima rentabilidad económica y comunicativa. La preocupación por comprender aspectos tales como saber cuál es o está siendo el futuro de la profesión periodística y cómo se están viendo alteradas las funciones del periodista y los métodos de producción son el eje sobre el que gira el trabajo de Esquivel Lozano (2010). Éste se plantea cuál es el estado de la cuestión del periodismo en estos momentos, basándose en la opinión y la reflexión de expertos reconocidos en el ámbito de la comunicación digital. Esta misma inquietud por la innovación en el terreno de la producción está presente en Fernández Beaumont (1999).

La transformación que acontece bajo la perspectiva documental es examinada por Marcos Recio, Sánchez Vigil y Serrada Gutiérrez (2009); Rubio Lacoba (2005, 2011) o García Jiménez (2002). En el primer caso los autores se basan en la metamorfosis que sufren los centros de documentación como uno de los elementos clave que interviene en la producción de las informaciones, así como en la labor desempeñada por sus profesionales en el ejercicio de su función. Para ello, toman como referencia algunos de los principales diarios americanos y europeos, con especial mención al caso español. Por su parte, el primer trabajo de la profesora Rubio Lacoba analiza el perfil periodístico, documental y tecnológico del documentalista en el medio digital, así como la interacción de sus funciones propias, con aquéllas inherentes al ámbito de la documentación informativa en general. En la disertación ofrecida en 2011, en el marco del IX Coloquio Internacional de Documentación, la autora establece las claves del cambio radical de las formas y funciones que se afianzan ante la consolidación de las versiones digitales de los diferentes medios ya existentes o aquellos nativos del entorno digital. Con ello, refuerza la idea reflejada en su anterior trabajo "Mejor que un buscador, un encontrador” (Rubio Lacoba y Blanco García, 2010).

Los trabajos de Caldera Serrano y Nuño Moral (2001); Nuño Moral (2003) y Acosta Valdés, Ramírez Céspedes y Marrero Santana (2011) centran su objetivo en el análisis descriptivo basado en la planificación y estructuración de los medios digitales. Mientras que los dos primeros se basan en una comparación realizada sobre los contenidos, secciones y servicios informativos que los principales periódicos españoles de información general le aportan al usuario, el último establece su valoración en periódicos tanto nacionales como internacionales. En esta misma línea, se encuentra el análisis desarrollado por el profesor Albornoz (2011), quien evalúa fundamentalmente los contenidos y 
recursos multimedia utilizados, la interactividad y las características de mercado.

Otros autores concentran sus esfuerzos exclusivamente en los productos y servicios presentes en los medios digitales. En este sentido sobresalen las investigaciones desarrolladas por González Quesada y Fuentes i Pujol (1998) o López Carreño y Pastor Sánchez (2010) sobre los denominados Servicios de Valor Añadido (SVA); López Aguirre (2009) centra el análisis tanto informativo como documental en la prensa digital mexicana, y Guallar (2011) adopta como eje de su trabajo los diferentes productos y servicios documentales para explicar la transformación a la que asiste la prensa desde esa perspectiva, resaltando el despunte vivido con la presencia de las hemerotecas digitales.

Por último, desde un punto de vista general, cabe subrayar los análisis generalizados de aspectos tales como visibilidad, hipertextualidad, usabilidad, calidad y las oportunidades que se encuentran en los diarios digitales. Al margen del trabajo presentado por Rodríguez Martínez, Codina y Pedraza-Jiménez $(2010,2012)$ sobre la interacción, comunicación y calidad (usabilidad, arquitectura de la información, accesibilidad), destaca el trabajo de Albornoz (2006) sobre el uso y funcionalidad del carácter hipertextual en el empleo de recursos audiovisuales y multimedia (audio, video y gráficos animados). En esta misma línea cabe mencionar la investigación de Guallar, Rovira y Ruíz (2010) en la cual se alude a los sistemas de acceso y de recuperación de dichos recursos.

Como advierte el lector, a pesar de la existencia de las referencias señaladas, pocos evalúan de manera exhaustiva el papel de las hemerotecas o archivos digitales. Sobre este aspecto concreto y determinante del trabajo presentado especificamos las siguientes reseñas.

Una de las evaluaciones pioneras en el campo de las hemerotecas digitales fue la presentada por Jiménez, González y Fuentes i Pujol (2000), donde advierten, por un lado, las características de este tipo de archivos en relación con las vías de acceso y consulta, visualización de recursos, fórmulas de interrogación y ayuda con que cuenta el usuario; por el otro, anotan los recursos informativos a los que el usuario accede en cada caso estudiado. Hay que subrayar el trabajo realizado por estos mismos autores en el año 2003, centrado en el acceso a la información audiovisual tanto de actualidad como retrospectiva. También de la profesora Jiménez López cabe mencionar dos trabajos posteriores. En el primero (2003) aborda el tema de la comercialización de los archivos digitales exponiendo los casos de $A B C$, El Periódico de Cataluña (en adelante El Periódico), Diario de Navarra, El Mundo, El País y La Vanguardia. En un segundo trabajo, titulado "Recursos electrónicos de información 
periodística: el caso de la prensa digital mexicana" (2004), la autora examina los servicios de acceso a toda la información contenida en el medio.

Al hilo de este último análisis resultan reseñables aquellos estudios de caso que se concretan en un diario o bien en una zona geográfica determinada, como es el caso de Sanz Calama (2003) sobre la hemeroteca digital de El País; o bien, los trabajos de Agirreazaldegi, Ronco y Camacho (2009) o el que estas mismas autoras realizan junto a Idoia Camacho y Carmen Peñafiel (2010). El primero de ellos analiza la situación de los medios impresos y audiovisuales en el País Vasco incluyendo agencias de noticias y grupos de comunicación, el segundo trata exclusivamente el contexto que caracteriza a los diarios digitales vascos. Por último referimos los trabajos de Martínez Rubio (2008, 2009), quien evalúa el sistema de recuperación que caracteriza a los diarios valencianos y andaluces respectivamente.

A raíz de las investigaciones existentes y tomando como referencia el citado artículo de Guallar y Abadal (2009), el objetivo principal de este trabajo es, por tanto, realizar un análisis descriptivo y cualitativo de las formas de acceso disponibles para el usuario en los sitios web de los diarios digitales de información general. La muestra se ha elaborado sobre los siguientes medios: El País, El Mundo, ABC, La Vanguardia, El Periódico, La Razón y Público.

Para conseguir tal propósito se ha tomado en cuenta el tipo de formatos a los que se puede acceder y cuáles son los sistemas de consulta en cada caso. De este último aspecto se lleva a cabo el estudio pormenorizado de cada buscador. En suma, los tres pilares básicos que vertebran este trabajo son: la tipología documental a la que se accede el método de acceso y, por último, las características de los archivos y sistemas de búsqueda.

\section{Metodología}

A continuación se representan los elementos que se han valorado en los diarios analizados y que constituyen el esquema completo de la investigación:

a) Tipología documental: los tipos de recursos documentales presentes en los diarios (noticias, gráficos, infografías, gráficos interactivos, fotogalerías, fotografías, videos, audios). En este análisis no se lleva a cabo un examen cuantitativo de los recursos multimedia existentes sino que se analiza la presencia de ellos como opción de consulta (localización y búsqueda de recursos multimedia, etiqueta o tag de acceso, etcétera).

b) Métodos de acceso: vías de consulta y visualización de los recursos documentales. 
c) Sistemas de recuperación: basados fundamentalmente en hemerotecas y búsquedas por palabras clave. De ellos, se analizan:

1. Denominación y localización.

2. Versiones disponibles y cobertura temporal.

3. Tipos de búsqueda y filtros de consulta.

4. Recursos recuperados: aspectos formales y relacionados con la aportación documental presente en los listados de documentos recuperados.

\section{RESULTADOS Y DISCUSIÓN}

\section{Tipología documental}

El formato textual continúa su hegemonía en los diarios digitales aunque los recursos multimedia cada vez estén más presentes. Tal y como se desprende del estudio, cuatro de los siete diarios analizados agrupan dichos recursos bajo la etiqueta "Multimedia" (ElMundo, ABC, El Periódico y La Razón); el resto cuenta con acceso directo a dichos documentos. Como se puede observar en la Tabla 1, los videos y fotografías están presentes en todos los diarios excepto La Razón, lo cual no quiere decir que dicho periódico no contenga fotografías, pero sí que no cuenta con un tag que nos lleve directamente a ellas.

Tabla 1. Tipos de recursos multimedia presentes en los diarios en línea

\begin{tabular}{|l|l|l|l|l|l|l|}
\hline \multicolumn{1}{|c|}{ El País } & \multicolumn{1}{c|}{ EI Mundo } & \multicolumn{1}{c|}{$A B C$} & La Vanguardia & El Periódico & \multicolumn{1}{c|}{ La Razón } & \multicolumn{1}{c|}{ Público } \\
\hline Videos & Videos & Videos & Videos & Videos & Videos & Videos \\
\hline Fotografías & Fotos & Galerías & Fotografías & Fotogalería & & Fotogalería \\
\hline Infografía & & & Infografía & & & \\
\hline & & Audios & & & & \\
\hline
\end{tabular}

En la muestra analizada los recursos multimedia no disponen directamente de un buscador propio. El acceso a los recursos se realiza a través de los sistemas generales, tal y como se aprecia más adelante. Lo que sí se observan son métodos de búsqueda simple, basada generalmente en las opciones de "Calendario" o por "Palabras clave", las cuales habilitan la localización de dichos materiales. La excepción la marca el diario La Razón, el cual no dispone de vías de acceso a los recursos multimedia, una vez consultado el sistema. Lo que muestra es un listado de títulos bajo la etiqueta "Videoteca".

El País, por ejemplo, posee un sistema de consulta propio al acceder a este recurso bajo la etiqueta "Infografía", y posibilita además llevar a cabo una acotación 
mediante los filtros "Sección" y "Calendario". El diario $A B C$, también dispone de buscador para las tres opciones, filtrando por "Formato" y "Sección”. El Periódico viabiliza la consulta tanto de videos como de fotografías, y puede restringir la recuperación a través del "Calendario" en ambos casos, y por "Temas" en las fotogalerías. Igualmente La Vanguardia dispone de buscador para videos y fotografías donde el usuario puede elegir, en este último caso, entre "Fotografías históricas", "Reportajes" y "Galerías fotográficas". Público cuenta con un buscador de videos limitando por "Sección". Por su parte, El Mundo realiza las consultas por palabra clave, filtrando por "Tipo de documento" (video o fotografías), una vez calculado el total. Y, por último, La Razón no dispone de ninguna herramienta individual para este tipo de recursos.

\section{Métodos de acceso}

Dejando al margen el acceso al contenido directamente a través de las propias noticias, fotografías, audios, videos o gráficos que aparecen en la primera página o portada del diario, su visualización mediante las diferentes secciones, o bien bajo la etiqueta Multimedia, tal y como se ha visto en el apartado anterior, los diarios en línea cuentan con otras vías de consulta para los diferentes recursos.

Tabla 2. Modos de acceso a los recursos informativos

\begin{tabular}{|c|c|c|c|c|c|c|c|c|}
\hline & El País & ElMundo & $A B C$ & La Vanguardia & El Periódico & La Razón & Público \\
\hline \multicolumn{2}{|c|}{ Ultimas Noticias/Hora } & $x$ & $x$ & $x$ & $x$ & & $x$ & \\
\hline \multicolumn{2}{|l|}{ Índice } & $x$ & & $x$ & $x$ & & & \\
\hline \multicolumn{2}{|c|}{ Lista normalizada } & $x$ & & $x$ & $x$ & & & \\
\hline \multirow{4}{*}{ "Lo más..." } & Visto/Leído & $x$ & $x$ & $x$ & $x$ & $x$ & $x$ & $x$ \\
\hline & Comentado & & & & $x$ & $x$ & & $x$ \\
\hline & \begin{tabular}{|l|} 
Valorado \\
\end{tabular} & & & & & $x$ & & $x$ \\
\hline & Enviado & & & & & $x$ & & \\
\hline \multicolumn{2}{|l|}{ Hemeroteca } & $x$ & $x$ & $x$ & $x$ & $x$ & $x$ & $x$ \\
\hline \multicolumn{2}{|c|}{$\begin{array}{l}\text { Sistema de búsqueda } \\
\text { (Caja de texto) }\end{array}$} & $x$ & $x$ & $x$ & $x$ & $x$ & $x$ & $x$ \\
\hline
\end{tabular}

Como refleja la Tabla 2, existen otras seis opciones para localizar los documentos que se demanden, y de ellas, "Lo más visto/leído", "Hemeroteca" y "búsqueda" por "Palabras clave", están presentes en todos los casos. Dada su complejidad comparativa y la necesidad de una explicación más detallada y exhaustiva, las dos últimas herramientas serán examinadas en el siguiente apartado.

Nos detenemos brevemente en algunas particularidades que deben tenerse en cuenta en las alternativas restantes. El servicio de "Última Hora" 
o "Últimas Noticias"1 está presente en todos los diarios excepto en El Periódico y Público, que junto con El Mundo tampoco disponen de acceso a través del "Índice". Este índice alfabético, temático y onomástico también es geográfico y de obras artísticas en el caso de $A B C$.

La opción denominada "Lista normalizada" consiste en destacar una media de cinco o seis temas relevantes de la actualidad periodística. Los tres periódicos que cuentan con esta opción lo hacen en la parte superior de la primera página del diario, bajo los epígrafes "Está pasando" en El País, "A fondo" en $A B C$ y, por último, "Destacamos" en La Vanguardia.

Una tercera vía permite visualizar los contenidos por las estadísticas propias de acceso de los usuarios a estos contenidos. De las cuatro opciones encontradas en el apartado "Lo más...", que cuenta con las cuatro posibilidades.

\section{Sistemas de recuperación}

Tal y como se ha mencionado anteriormente, "Hemeroteca" y "Buscador" (o "Palabras clave") son los dos instrumentos de consulta más importantes a la hora de acceder al contenido, los cuales están presentes en toda la muestra analizada. Con objeto de conseguir una mayor inteligibilidad, se expone un análisis descriptivo y comparativo, que resultó de la evaluación realizada en el apartado "Metodología" del presente trabajo.

1. Denominación y localización

Todos los diarios optan por la etiqueta "Hemeroteca" excepto El Periódico, que utiliza la de "Archivo". Tanto en este diario como en La Razón su acceso es de pago, ya que aunque el primero permita hacer la consulta la visualización completa del contenido no es gratuita.

Incluso El País distingue entre "Hemeroteca" y "Archivo papel". La diferencia estriba en que en la primera, la consulta se realiza únicamente mediante el "Calendario" (año, mes, día, franja horaria) y alberga sólo los últimos dos meses. Por su parte, en el "Archivo papel”, la consulta se puede realizar también por "Palabras clave"(siguiendo las mismas características que en la opción "Buscador", que se explicará más adelante), pero en este caso, no se almacenan los dos últimos meses.

1 Ambas opciones se han considerado sinónimas aunque, por ejemplo, $A B C$ diferencia de forma clara entre ambas en función de la procedencia del recurso. Así, la etiqueta "Última hora" incluye las últimas noticias de agencias, mientras que bajo el epígrafe "Últimas noticias" recoge las informaciones del periódico. 
Público y El Mundo equiparan la actividad de la "Hemeroteca" a la consulta por "Palabras clave", aunque el primero cuente con esta segunda opción para realizar las consultas en un buscador externo. Es decir, mientras que Público utiliza la etiqueta "Hemeroteca" para hacer las consultas internas y el "Buscador" para realizar las consultas, en Google, el periódico El Mundo permite, desde ambas herramientas, seleccionar dónde realizar la consulta de manera que, en este caso, se considerarían sinónimas. Por último, $A B C$ y La Vanguardia son los ejemplos que, desde el punto de vista informativo y visual, diferencian más entre ambas herramientas. Un elemento que los une es el marcado carácter histórico o retrospectivo que se deriva cuando se accede a la hemeroteca de ambos. De hecho, los dos disponen de la opción denominada "Un día en tu vida" para realizar la consulta de una fecha concreta, desde 1881 en La Vanguardia o desde 1891 en el caso de $A B C,{ }^{2}$ si dicha consulta se realiza en la revista Blanco y Negro. En la hemeroteca de La Vanguardia, destaca la opción "Tendencias”, que permite visualizar gráficamente el tratamiento cuantitativo que sobre ese tema se ha realizado en el periódico a lo largo del tiempo.

Para finalizar cabe resaltar que a excepción de Público, el cual presenta un listado de titulares organizados en secciones cuando accedemos a la "Hemeroteca", los restantes diarios disponen de la opción de buscar por "Palabras clave", teniendo en cuenta la necesidad de suscripción aludida en El Periódico o la limitación temporal mencionada en El País, la cual está también vigente en $A B C$. Esto es, la consulta a través de palabra clave en la hemeroteca de este último diario no incluye los últimos quince días en curso.

En cuanto a la localización, la muestra analizada presenta ambas herramientas, "Hemeroteca"y "Buscador", en la primera página o portada de los diarios analizados.

2. Versiones y cobertura temporal

El País, ABC y La Vanguardia permiten una doble alternativa dado que hacen posible visualizar tanto la versión en línea como la impresa. La cobertura temporal se remonta al año 1976, 1903 o 1881 respectivamente. $A B C$ dispone de documentos desde 1891 pero corresponden a informaciones publicadas en la revista Blanco y Negro. El resto de periódicos remontan sus fechas de consulta al año 2000 o 2001, en el caso de El Mundo y El Periódico. Por su parte, Público lo hace desde su aparición en el año 2007. Estos últimos, junto con La Razón, permiten el acceso gratuito a la edición digital. 
3. Tipo de búsqueda y filtros de consulta Excepto La Razón y Público, el resto de ejemplos cuenta con una doble posibilidad de búsqueda: "simple" y "avanzada". En estos dos casos sólo existe la búsqueda simple por palabras clave a través de una única caja de texto y no hay posibilidad de filtrar la consulta. En el caso de La Razón, ésta se lleva a cabo no sólo en el diario sino también en un navegador externo, que ofrece documentos procedentes de fuentes externas. Por su parte, Público utiliza la tecnología del navegador Google, pero los resultados que se obtienen son documentos y recursos elaborados por el propio medio.

Se puede afirmar que en ningún caso, a excepción de El País, se cuenta con un acceso directo a la consulta avanzada. Es decir, este nivel de limitación o acotación está presente una vez que se le ha formulado la interrogación al sistema en la caja de texto correspondiente a la búsqueda simple. A posteriori, conocida la organización y estructura interna del periódico, ${ }^{4}$ se puede testificar que El País sí presenta el acceso directo a la búsqueda Avanzada a través de la etiqueta Archivo web. Por su parte, la consulta simple se realizaría a través de la caja de texto situada en la parte superior de la primera página del periódico.

El resto de la muestra revela las siguientes particularidades opcionales en sus sistemas de búsqueda mediante "Palabras clave", tal y como se refleja en la Tabla 3 (página siguiente).

De forma breve se detallan algunos aspectos relevantes para su comprensión. En primer lugar se diferencia entre "Cobertura temporal" y "Fechas". La primera denominación hace referencia a la oportunidad de elegir entre periodos de tiempo ya cerrados ("Última semana", "Último mes", "Último año"; o bien, especificar la búsqueda en un año concreto). Mientras, la etiqueta "Fecha" le permite al usuario marcar una jornada concreta (día, mes, año), o bien, establecer un rango temporal de búsqueda (desde-hasta).

La acotación de registros mediante lenguajes de consulta está presente en $A B C$ y La Vanguardia. ${ }^{5}$ El primero permite el uso de los operadores lógi$\cos (\mathrm{AND} / \mathrm{OR} / \mathrm{NOT})$ y la redacción de frases exactas como elemento de recuperación, mientras que el segundo sólo habilita los operadores AND y OR, junto con la restricción por frase.

3 Se opta por utilizar ambas etiquetas con el objeto de unificar la comprensión de la casuística existente.

4 Esto es, el usuario requiere conocer el sistema de organización y etiquetado utilizado por el diario para constatar la posibilidad del doble acceso al que se está haciendo referencia.

5 Cabe resaltar además, en el caso de La Vanguardia, que en la "Hemeroteca" el usuario puede acotar la consulta por medio de tres vías: "Edición" o "Suplemento", lenguaje de consulta mediante el operador de exclusión (OR) y, por último, indicando la visualización de portadas exclusivamente. 
Tabla 3. Filtros de acotación en los Sistemas de búsqueda Simple y Avanzada

\begin{tabular}{|c|c|c|c|c|c|c|c|}
\hline & El País & El Mundo & $A B C$ & La Vanguardia & El Periódico & La Razón & Público \\
\hline \multirow{9}{*}{$\begin{array}{l}\text { Búsqueda } \\
\text { Simple }\end{array}$} & & \begin{tabular}{|l} 
Búsqueda \\
interna o en \\
otras fuentes
\end{tabular} & & & & $\begin{array}{l}\text { Búsqueda } \\
\text { interna o } \\
\text { en otras } \\
\text { fuentes } \\
\end{array}$ & \\
\hline & & $\begin{array}{l}\text { Tipo } \\
\text { documental }\end{array}$ & $\begin{array}{l}\text { Tipo } \\
\text { documental }\end{array}$ & & \begin{tabular}{|l} 
Tipo \\
documental
\end{tabular} & & \\
\hline & & Fecha & Fecha & & & & \\
\hline & & Sección & Sección & & & & \\
\hline & & Suplementos & & & & & \\
\hline & & $\begin{array}{l}\text { Cobertura } \\
\text { temporal }\end{array}$ & $\begin{array}{l}\text { Cobertura } \\
\text { temporal }\end{array}$ & & & & \\
\hline & & & $\begin{array}{l}\text { Versión } \\
\text { impresa o } \\
\text { digital }\end{array}$ & & & & \\
\hline & & & Edición & & & & \\
\hline & & & & & $\begin{array}{l}\text { Usar la } \\
\text { tecnología } \\
\text { de Google }\end{array}$ & & $\begin{array}{l}\text { Usar la } \\
\text { tecnología } \\
\text { de Google }\end{array}$ \\
\hline \multirow{6}{*}{$\begin{array}{l}\text { Búsqueda } \\
\text { Avanzada }\end{array}$} & Fecha & $\begin{array}{l}\text { Fecha/ } \\
\text { Rango }\end{array}$ & & $\begin{array}{l}\text { Fecha/ } \\
\text { Rango }\end{array}$ & $\begin{array}{l}\text { Fecha/ } \\
\text { Rango }\end{array}$ & & \\
\hline & $\begin{array}{l}\text { Tipo } \\
\text { documental }\end{array}$ & & & $\begin{array}{l}\text { Tipo } \\
\text { documental }\end{array}$ & & & \\
\hline & & & $\begin{array}{l}\text { Lenguaje } \\
\text { de consulta }\end{array}$ & \begin{tabular}{|l} 
Lenguaje \\
de consulta
\end{tabular} & & & \\
\hline & & & & Autor & & & \\
\hline & & Relevancia & & & & & \\
\hline & & & & Sección & Sección & & \\
\hline
\end{tabular}

El Periódico y Público disponen del uso de la tecnología de Google para realizar las consultas. La diferencia entre ambos estriba en que, en el segundo caso, se convierte en la única vía de acceso a los recursos, mientras que en el primero se posibilita la entrada a una búsqueda interna o propia. Por último es posible mencionar que los filtros de "Sección" y "Edición" de $A B C$, así como los de "Fecha", "Sección" y "Suplementos" de El Mun$d o$, nos aportan la información del número de documentos correspondiente con cada una de esas alternativas. Obviamente este dato es de interés en el siguiente apartado.

4. Recursos recuperados

De los documentos recuperados, los resultados obtenidos se presentan estructurados en dos áreas: aspectos formales y aportación documental. 
Aspectos formales

Dentro de este bloque se incluyen cuatro parámetros: ${ }^{6}$

- Ordenación de resultados

A excepción de Público, cuyos resultados aparecen clasificados por secciones, el resto de los diarios siguen los criterios de ordenación por "Fecha" y "Relevancia". Además, en el caso de $A B C$ hay que señalar que el criterio "Relevancia" aparece en unión al de "Fecha" y no viceversa.

- Visualización de número de documentos por página El Mundo y $A B C$ permiten al usuario determinar el rango de documentos que quiere visualizar por página a partir del cómputo total resultante.

- Visualizar la edición impresa o digital

Opción que se encuentra por defecto cuando se accede a El País y que tendrá que especificar el usuario cuando formule la consulta en $A B C$. Además, para este segundo diario ha de tenerse en cuenta que en el caso de la edición impresa, el sistema de búsqueda no contiene los acontecimientos sucedidos en los últimos quince días en curso.

- Número total de documentos resultantes y cuantificación por tipología

Esta alternativa no consiste en valorar si el sistema permite internamente filtrar por tipo de documentos. Su razón de ser reside en evaluar si, cuando se obtienen los registros tras la interrogación previa al sistema, éste nos indica el valor numérico del total de recursos obtenidos y se especifica su distribución, conforme a la tipología documental establecida por cada medio. ${ }^{7}$

Aportación documental

En este segundo bloque se evalúan ítems tales como:

- Datos aportados por cada documento

En la Tabla 4 se presenta la comparación del análisis llevado a cabo sobre la página de resultados tras la formulación de la búsqueda al sistema. Se excluye el periódico Público dado que las consultas las realiza utilizando exclusivamente la tecnología de Google, y el único dato presente en todos sus registros es la URL

6 En este apartado se han excluido las opciones de "Gestión" (comentar, imprimir, etc.) definidas por los profesores Guallar y Abadal dado que ningún diario dispone de dicha posibilidad en las páginas de resultados obtenidas.

7 Conviene recordar en este apartado la opción de este periódico junto con el diario $A B C$, cuando se explicaba que en los resultados se mencionaba el número de documentos y recursos disponibles en función de los filtros de "Sección", "Edición”, "Fecha” y "Suplementos". 
que lleva directamente al recurso interno. Para el resto de diarios, el resultado sería el siguiente:

Tabla 4. Información aportada en las listas de resultados ${ }^{8}$

\begin{tabular}{|l|l|l|l|l|l|}
\hline \multicolumn{1}{|c|}{ EI País } & \multicolumn{1}{c|}{ El Mundo } & \multicolumn{1}{c|}{ ABC } & La Vanguardia & \multicolumn{1}{c|}{ El Periódico } & \multicolumn{1}{c|}{ La Razón } \\
\hline Fecha & Fecha & Fecha & Fecha & Fecha & Fecha \\
\hline Relevancia & Relevancia & & & & \\
\hline & Antetítulo & & & & \\
\hline Título & Título & Título & Título & Título & Título \\
\hline Subtítulo & Subtítulo & Subtítulo & Subtítulo & Subtítulo & Subtítulo \\
\hline Autor & Autor & Autor & Autor & Autor & \\
\hline & Tipo documental & & & & \\
\hline & & Sección & Sección & Sección & \\
\hline & &
\end{tabular}

Tal y como se observa, los dos extremos los ocupan La Razón y El Mundo. Destaca el hecho de que ABC, La Vanguardia y El Periódico faciliten al usuario los mismos datos por documento. En el indicador "Subtítulo" se incluye a La Vanguardia que, por lo general, se corresponde con el inicio del cuerpo del texto.

- Enlace a recursos relacionados

El País y El Mundo son los únicos ejemplos que se han encontrado donde se muestra una relación con documentos relacionados según la consulta establecida. Obviamente se valora el enlace desde la página de resultados, no individualmente, una vez que el usuario accede a cada uno de dichos registros.

- Ayuda en la consulta desde el punto de vista temático Al igual que en el punto anterior los periódicos antes mencionados son los únicos que ofrecen una ayuda desde la perspectiva del contenido. Hay que tener en cuenta que este parámetro difiere de otras formas de acceso establecidas en la Tabla 2, bajo las denominaciones de "Índice" o "Lista normalizada".

En este caso se trata de evaluar la presencia de términos (temáticos, onomásticos o geográficos) que puedan estar relacionados con los conceptos empleados por el usuario en la consulta, de manera que le permitan concretar su demanda. Mientras que El Mundo presenta un listado de términos relacionados con

8 En este caso se excluye si la página de resultados lleva adjunta la existencia de fotografías y videos, ya que tal y como se ha comprobado y en ocasiones aparece una fotografía y el recurso que realmente está presente en dicho documento es un video. Estos casos se han encontrado en La Vanguardia y Público, por ejemplo. 
la consulta general llevada a cabo, El País dispone dicha enumeración para cada uno de los resultados.

\section{Conclusiones}

En relación a los tres bloques evaluados, las principales conclusiones se vertebran en dos aspectos. En primer lugar, la multimedialidad posibilita que los diarios digitales hayan superado las barreras heredadas del formato impreso consiguiendo así equiparase al medio televisivo, el cual, en su origen analógico, ya disponía de los recursos fundamentales: texto, audio, fotografías y videos.

Todo ello ha situado a las hemerotecas digitales como una fuente de información única y excepcional tanto económica como culturalmente. En comparación con estudios realizados con anterioridad y que se han reseñado en la bibliografía, se aprecia un incremento de la presencia de los recursos multimedia desde la perspectiva informativa, a pesar de la existencia de lagunas importantes, tal y como ha quedado reflejado en La Razón.

En segundo lugar, junto a los sistemas de búsqueda tradicionales cada vez son más numerosas las posibilidades con que cuenta el usuario al momento de acceder a los contenidos. Destacan El País, ABC y La Vanguardia al disponer de las seis alternativas desarrolladas en el apartado "Métodos de acceso" del presente trabajo.

Sería recomendable que los medios digitales, al margen de la rentabilidad económica que los sistemas de búsqueda puedan suponer para ellos, determinaran la planificación de sus hemerotecas en función del usuario final. Es en este apartado donde se ha encontrado un mayor vacío y, como consecuencia, donde se precisa de una mayor atención y mayores cambios, con el objeto de que los recursos contenidos en ellos resulten útiles y disponibles.

Aspectos tales como indicar de manera explícita la cobertura temporal del fondo digital o la versión (digital o impresa) sobre la que se realiza la consulta; evitar la duplicación innecesaria de etiquetas sinónimas o especificar, de manera exacta, el recurso multimedia que acompaña a la información textual aportada en las páginas de resultados son sin duda, aspectos que deben someterse a revisión y mejorar así los sitios web de los medios de comunicación en aras de una mayor eficacia y efectividad. 


\section{Bibliografía}

Acosta Valdés, Misleiny; Ramírez Céspedes, Zulia; Marrero Santana, Liliam (2011), "Organización de la información en sitios web periodísticos”, en El Profesional de la Información, vol. 20, núm. 1, pp. 54-60.

Agirreazaldegi, Teresa; Ronco, Milagros; Camacho, Idoia (2009), "El desarrollo de la documentación en los medios de comunicación del País Vasco", en XI Jornadas Españolas de Documentación, Zaragoza, pp. 175-182.

Albornoz, Luis Alfonso (2006), "Los principales diarios online en español: el empleo de recursos audiovisuales y multimedia, e hipervínculos", en Telos: Cuadernos de comunicación e innovación, núm. 66, pp. 23-30.

_ (2011), "Prensa digital: características de los principales diarios online en español", en Revista Latinoamericana de Ciencias de la Comunicación", disponible en: http://www.eca.usp.br/associa/alaic/ revista/r4/ccientifica_02.pdf

Álvarez Marcos, José; Parra Valcarce, David (2004), Ciberperiodismo: periodismo especializado, Madrid: Síntesis, ISBN: 978-84-9756-218-8.

Camacho, Idoia; Agirreazaldegi, Teresa; Ronco, Milagros; Peñafiel, Carmen (2010), “¿Evolución o revolución?: adaptación de los servicios de documentación a la era digital”, en II Congreso Internacional AE-IC, disponible en: http://www.aeic2010malaga.org/upload /ok/243.pdf

Caldera Serrano, Jorge; Nuño Moral, María Victoria (2001), "Análisis comparativo de las web de prensa española en Internet", en Documentación de las ciencias de la información, núm. 24, pp. 195-211.

Esquivel Lozano, Fernando (2010), "Los retos del periodismo digital", en II Congreso Internacional AE-IC, disponible en: http://www. aeic2010malaga.org/upload/ok/187.pdf

Fernández Beaumont, José (1999), "La producción de la información en la era digital: el periodismo español en Internet”, en La liberalización de las telecomunicaciones en un mundo global, pp. 499-512, ISBN: 978-84-7695-632-8.

García Jiménez, Antonio (2002), Organización y gestión del conocimiento en la comunicación, Gijón: Trea, ISBN: 97884970-40-464.

García Jiménez, Antonio; Rupérez Rubio, Paloma (2007), Aproximaciones al periodismo digital, Madrid: Universidad Rey Juan Carlos: Universidad Rey Juan Carlos, Dykinson. ISBN: 978-84-9849-140-1.

García-Alonso Montoya, Pedro (2010), El periodismo digital en la empresa informativa, disponible en: http://www.cibersociedad.net/ congres2009/actes/html/com_el-periodismo-digital-en-la-empre sa-informativa_807.html

González Quesada, Alfonso; Fuentes i Pujol, Eulalia (1998), "La prensa española en Internet: análisis de los servicios de valor añadido", en Jornadas Españolas de Documentación, pp. 281-292. 
Guallar, Javier (2011), "La documentación en la prensa digital: nuevas tendencias y perspectivas", en III Congreso Internacional de Ciberperiodismo y web 2.0, disponible en: http://eprints.rclis.org/16326/1/ ciberpebi2011_guallar_documentacion \%20prensa\%20digital.pdf -; Abadal, E. (2009), "La evaluación de hemerotecas de prensa digital: indicadores y ejemplos de buenas prácticas”, en El Profesional de la Información, vol. 18, núm. 3, pp. 255-269.

—_ Rovira, Cristòfol; Ruíz, Sara (2010), "Multimedialidad en la prensa digital: elementos multimedia y sistemas de recuperación en los principales diarios digitales españoles", en El Profesional de la Información, vol. 19 núm. 6, pp. 620-629.

Jiménez López, Angels (2003), "Hemerotecas de pago en la prensa digital española”, en El Profesional de la Información, vol. 12, núm. 6, pp. 473-474.

— (2004), "Recursos electrónicos de información periodística: el caso de la prensa digital mexicana”, en XXII Coloquio de Investigación Bibliotecológica, pp. 79-93.

Jiménez, Angels; Fuentes i Pujol, Eulalia; González, Alfons (2003), "Los archivos audiovisuales en los medios de comunicación digital”, en Scire, vol. 9, núm. 2, pp. 99-110.

__; González, Alfons; Fuentes i Pujol, Eulalia (2000), "Las hemerotecas digitales de la prensa en Internet”, en El Profesional de la Información, vol. 9, núm. 5, pp. 15-24.

López Aguirre, José L. (2009), "Estudio de servicios y productos ciberdocumentales en la prensa digital mexicana", en Documentación de las Ciencias de la información, vol. 32, pp. 93-132.

López Carreño, Rosana; Pastor Sánchez, Juan Antonio (2010), “Actualización del modelo de portal periodístico de prensa española”, en Anales de Documentación, vol. 13, pp. 177-184.

Luján Zavala, Juan Carlos (2003), "Periodismo digital y la relación con los usuarios en la era digital”, en Razón y Palabra, disponible en: http://www.razonypalabra.org.mx/anteriores/n30/jlujan.html

Marcos Recio, Juan C. (2005), "Una década de periódicos en Internet: estrategias documentales”, en Scire, vol. 11, núm. 2, pp. 63-77 -; Sánchez Vigil, Juan M.; Serrada Gutiérrez, María (2009), "Nuevos paradigmas periodísticos y documentales en los periódicos digitales: estudio de casos en España”, en Investigación Bibliotecológica, vol. 23, núm. 49, pp. 43-65.

Martín Herrera, Inma (2010), "Prensa electrónica: cómo hacer periodismo en la era digital”, disponible en: http://www.cibersociedad. net/congres2009/ca/coms/prensa-electronica-como-hacer-periodismo-en-la-era-digital/499/

Martínez Rubio, Rosa (2008), "La recuperación de la información en los periódicos digitales valencianos", en Comunicación local y nuevos formatos periodísticos en Internet: cibermedios, confidenciales y weblogs, disponible en: http://www.cibermediosvalencianos.es/ comloc/Martinez.pdf 
_ (2009), "Estructura de la información y su recuperación en los diarios digitales andaluces: análisis de las vías de comunicación interna”, en II Congreso Internacional AE-IC, disponible en: http:// www.aeic2010malaga.org/upload/ok/443.pdf

Nuño Moral, María Victoria (2003), “Organización de los web site de periódicos españoles en Internet”, disponible en http://www.upf. edu/hipertextnet/numero-1/periodicos.html

Palacios, Marcos; Díaz Noci, Javier (2009), Online Journalism: research methods. A multidisciplinary approach in comparative perspective, disponible en http:/www.ehu.es/argitalpenak/images/ stories/libros_gratuitos_en_pdf/Ciencias_Sociales/Online $\% 20$ Journalism\%20Research\%20methods.pdf

Pareja Pérez, Víctor Manuel (2003), "Introducción al periodismo digital", en Guía de Internet para periodistas, Madrid: Centro de Información y Documentación Científica, pp. 29-58, ISBN: 84-0008092-0

(2006), "Periodismo digital. Medios de comunicación en Internet", en La información especializada en Internet: directorio de recursos de interés académico y profesional, Madrid: Centro de Información y Documentación Científica, pp. 237-276, ISBN: 84-0008436-5

Parra Valcarce, David; Edo, Concha; Flores, Jesús; García-Alonso, Pedro; Marcos Recio, Juan C. (2008), "Proceso de transformación de los cibermedios: los retos de las empresas periodísticas", en Revista Latina de Comunicación Social, núm. 63, disponible en: http://www.ull.es/publicaciones/latina/_2008/06_Concha_Edo. html

Rodríguez-Martínez, Ruth; Codina, Lluis; Pedraza-Jiménez, Rafael (2010), "Cibermedios y Web 2.0: modelo de análisis y resultados de aplicación”, en El Profesional de la Información, vol. 19, núm. 1, pp. 35-44.

(2012), "Indicadores para la evaluación de la calidad en cibermedios: análisis de la interacción y de la adopción de la Web 2.0", en Revista Española de Documentación Cientifica, vol. 35, núm. 1, pp. 61-93.

Rubio Lacoba, María (2005), "Nuevos tiempos para la documentación informativa en el periodismo digital: viejas y nuevas funciones del servicio de documentación digital”, en Comunicación y Sociedad, vol. 18, núm. 1, pp. 153-168.

_ (2011), "El futuro del documentalista en los medios digitales", en IX Coloquio Internacional de Documentación, disponible en: http: //campus.usal.es/ coloquiobyd/Rubio_ColoquioSalamanca2011. pdf

_- B Banco García, Juan Carlos (2010), "Mejor que un buscador, un encontrador", en Documentación de Ciencias de la Información, vol. 33, pp. 273-287. 
Salaverría, Ramón (2005), Cibermedios: el impacto de Internet en los medios de comunicación en España, Sevilla: Comunicación Social Ediciones y Publicaciones, ISBN: 84-96082-33-4

Sanz Calama, Flora (2003), "La hemeroteca digital de El País", en IV Jornadas de Bibliotecas digitales, pp. 135-144.

$\infty$ 